\title{
COMPETENCE PROFILING IN VIRTUAL COMPANIES
}

\begin{abstract}
Barbara Odenthal
Chair and Institute of Industrial Engineering and Ergonomics (IAW) at University of Technology Aachen (RWTH Aachen University), GERMANY

e-mail:B.Odenthal@iaw.rwth-aachen.de
\end{abstract}

Meikel Peters

Chair and Institute of Industrial Engineering and Ergonomics (IAW) at University of Technology Aachen (RWTH Aachen University), GERMANY

e-mail:M.Peters@iaw.rwth-aachen.de

\begin{abstract}
The reorganization of the supply chain in the aerospace industry has led to a new situation for small and medium-sized enterprises (SME). In order to react to the changed requirements, one possible solution is the project-oriented and temporary cooperation of SME by building a Virtual Company. This again presents the employees with new challenges regarding the work requirements. Within the research project AerViCo - Aerospace Virtual Company - special tools and instruments considering the effects on employee performance behavior are being developed in order to increase labor productivity and to support the employees within the cooperation.
\end{abstract}

\section{INTRODUCTION}

During the past years the development of the corporate strategy of manufacturers in the aerospace industry has led to a new situation for small and medium-sized enterprises (SME). In order to lower their costs, European consolidated companies in the aerospace industry (Airbus, EADS, astrium, Lagardere, ...) have re-organized their supplier network. The number of direct suppliers, that have to be audited and certified, was reduced from several hundred to less than ten, which leads to a significant decrease of administrative work for the large enterprises (figure 1).
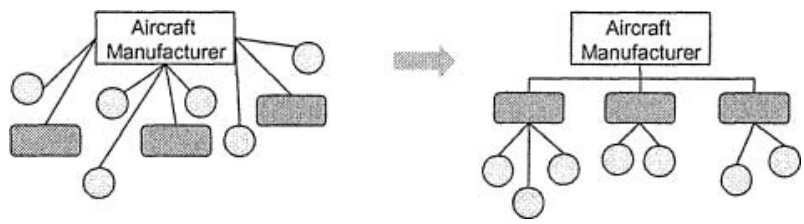

Fig. 1: Re-organization of the supplier network

In consequence, the strategic suppliers are commissioned to deliver completed systems instead of components and assemblies. 
In addition to the order processing, the first level suppliers are now also responsible for the monitoring and the compliance of second and third level suppliers with the quality standards. Consequently the above mentioned requirements are handed on to the second and the third level suppliers which are mainly SME. In order to respond to the new situation, one possible solution is the creation of a virtual network of SME which join forces in the case of an order processing and adjust flexibly to the requirements at hand (Peters/Bernhard, 2004). Because the success of interorganizational and knowledge-intensive cooperation in a virtual context depends to a large extent on the efficient and effective interaction of employees, the employees of the SME are confronted with new challenges. In order to make work more efficient and to support the employees within the cooperation, it is necessary to provide special tools and instruments considering the effects on employee performance behavior.

In order to ensure compliance of employee behavior while working and acting in cooperation with the Virtual Companies' (VC) goals three aspects are of importance (Killich/Peters, 2003): commitment (motivation), capability (competencies) and conditions (influence of organization, leadership and culture), see figure 2.

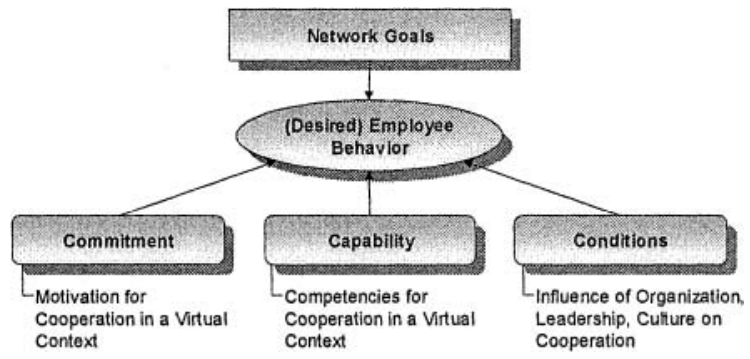

Fig. 2: Employee-Related Aspects of Virtual Companies

In order to support the SME with the operation of a VC, the research project AerViCo - Aerospace Virtual Company - was initiated. The objective of the project is to develop a cooperation network of SME which is the basis for forming project consortiums to process a certain order. One of the achievements of the project is the development of a method to support the employees' competencies for cooperation in a virtual context in order to enable them to act in accordance with the network goals. Even in a virtual and networked context social competencies, language and communication skills become more and more important and are needed in addition to excellent professional competencies. Therefore, in this project a method for profiling and enhancing employee competencies is being developed.

\section{CURRENT STATE}

In order to acquire a survey of the current state of cooperation within the aerospace industry, interviews (face-to-face and via questionnaires) were carried out (Odenthal/Peters, 2005), in which different fields of interest regarding the job design could be identified. On closer examination the role of the employees in a Virtual 
Company must be differentiated into the employees who are directly involved in the cooperation and those who are not. In the context of the project AerViCo the companies concerned mainly offer development, construction and testing services. In these knowledge-intensive services the employees are directly involved in the cooperation. That means that there are points of contact between the company (project leader and member of the project team) and the client. Because of this type of work, special job requirements for the employees involved directly in the cooperation occur. Professional and linguistic competencies, high flexibility in terms of the processing of different tasks and social competencies are necessary in order to successfully operate in interorganizational projects. Furthermore, different types of organizational culture in the virtual context lead to specific characteristics in respect to the job conditions: different ways of working, decision structures, authorities of the contact persons, different remuneration and differences concerning the influence of the workers' council hold the danger of conflicts.

On the one hand companies expect their employees to fulfill these requirements, but on the other hand SME so far hardly provide systematical and methodical support and development of the employees' competencies. Often the companies employ staff members which develop "on-the-job" adapting to the postulated requirements. This procedure holds the danger of not making use of the full potentials concerning employee competencies.

\section{TARGET COMPETENCE PROFILES IN VIRTUAL COMPANIES}

In order to identify, support and develop the competencies of the employees involved in the cooperation, it is crucial to identify the target competence profile for the concerned roles within a VC which can be consulted in order to compare the target competence profile with the actual competence profile of the employee. This comparison of the two profiles offers the possibility of systematically implementing measures of competence development.

One of the achievements of the AerViCo-project is the development of a method which generates target competence profiles for workplaces and activity fields of employees in SME while considering the special requirements in a VC.

\subsection{A method for generating target competence profiles}

The method for generating target competence profiles follows a synthetic approach. The basic idea behind the method is that the work tasks and work situation of a role in a virtual company can be described by a set of activities and activity features. Furthermore, activities and activity features require corresponding competencies of the acting person in order to successfully carry out the work tasks.

Following this idea, a classification of activities and activity features was developed on the basis of existing models of activities (Stahl 1998; Kabel 2001) and an analysis of existing methods of psychological job evaluation (Richter 2001). This classification allows the description of work contents and work situations in a virtual 
context. Furthermore, the described method is based on an existing classification of competencies (Erpenbeck et al. 2002). Erpenbeck defines competencies as dispositions of self-organization, which can be divided in four parts (see figure 3):

- personal competence: Disposition to act reflexive self-organized

- activity and acting competence: Disposition to act holistic self-organized

- professional and methodical competence: Disposition to act theoreticalmethodical self-organized

- social-communicative competence: Disposition to act communicative and cooperative self-organized

While the classification of competencies is universally valid and not tailored for inter-organizational cooperation, the classification of activities and activity features allows for the specific aspects of work in a virtual context. For instance, concerning activities and activity features, working in VCs includes cooperation processes on a personal level and a high flexibility of the working situation in terms of working time, working place and information flows. These characteristics were taken into account for the development of the classification of the activities and activity features (see figure 3). In order to prove the completeness of this classification consisting of 29 activities and 21 activity features - the classification was used to represent several existing work processes and work task descriptions with a cooperative character.
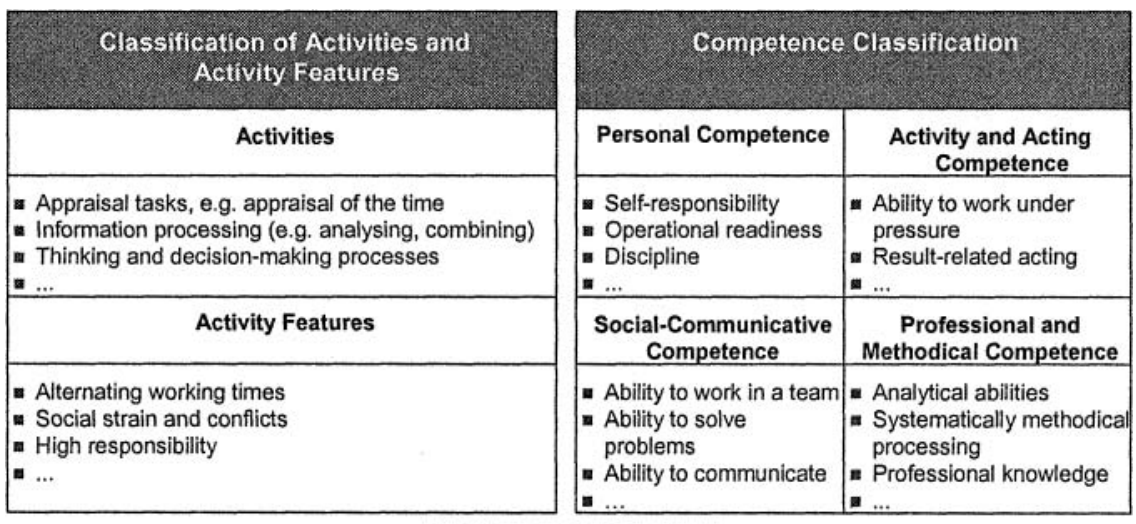

General Allocation of Competencies to Activities and Activity Features

Fig. 3: General allocation of competencies to activities and activity features

Using the described classifications, the basis of the method is formed by a weighted allocation of competencies to activities and activity features which was developed in workshops with experts from the field of cooperation in the aerospace industry. However, the goal is to establish a connection between the work tasks of a role in the $\mathrm{VC}$ and the required competencies. This happens by describing specific work tasks and situations using the defined activities and activity features. With the predefined allocation of competencies to activities and activity features this leads directly to the respective target competence profile of the considered role. To briefly 
explain this train of thought, the Prime Contractor of the network is taken as an example. By working in a VC, "cooperative problem solving" (activity) gains in importance. Moreover, the job of a Prime Contractor involves frequent travelling (activity feature). Both aspects lead to a higher importance of social-communicative competencies and stress capacity.

\subsection{Application of the method considering as example the role of the Broker}

In the following, the application of the procedure of generating a target competence profile will be shown using the role of the Broker of the VC as example.

The tasks of a broker are characterized as follows. He/she is responsible for coordination and administrative activities on the platform of the cooperation network independent of the concrete order processing. This includes on the one hand tasks of configuring a cooperation network, e.g. legal agreements between the network partners and the development of the IT-infrastructure for the network. On the other hand, the broker must ensure the smooth flow and operation of the cooperation network by taking over the task of the administration of the partners, conflict management and the controlling of the platform. Additionally, he/she is responsible for the marketing of the cooperation network. The broker acts as a mediator between the customer and the network by marketing the products or services of the network and by forwarding costumers inquiries to the network partners.

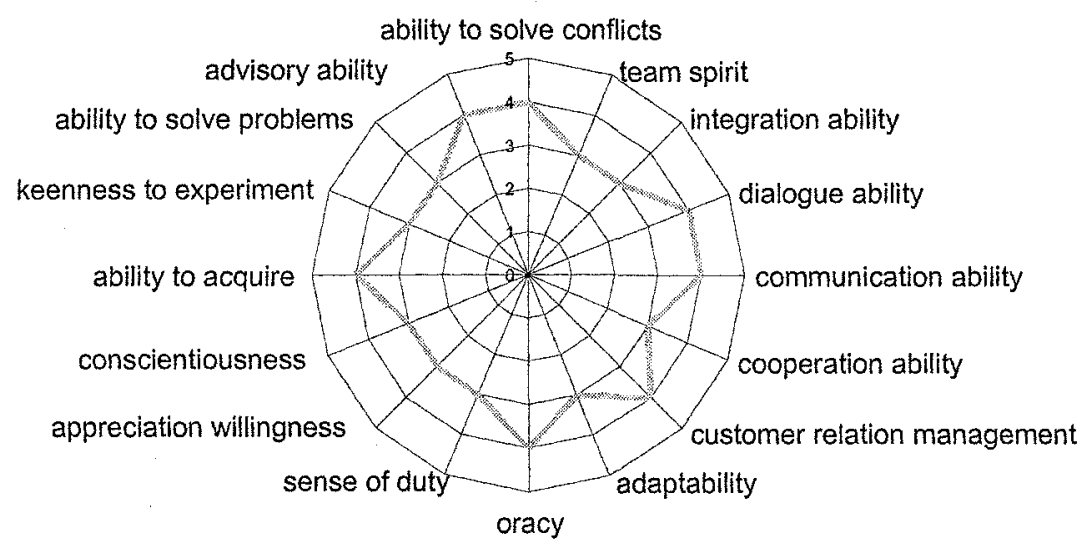

Fig. 4: Example of a target competence profile of the broker of a VC (extract: social-communicative competence)

Using the developed method, it is now possible to realise a target competence profile of a broker by allocating and weighting activities and activity features to the job tasks. This step is carried out by the user e.g. the project leader in order to fill a vacancy. The allocation of required competencies to the activities and features is implicitly given by the method. Figure 4 shows an extract of an example for a target competence profile of a broker of a VC. From the point of view of the enterprises, this method for creating target profiles facilitates the process of selection of personnel by means of comparing the target profile of the work field and the current 
state profile of the (potential) employee. From the point of view of the employee, the comparison of the target profile and the current state profile establishes and provides a basis for initiating measures for personnel development in order to increase the competencies and thus his/her capability continuously.

\subsection{Current state profiles and competence development measures}

In order to complete the competence profiling, it is necessary to measure the existing competencies of the employees involved in the cooperation (current state competence profile). Because of the wide range of existing competence measuring instruments the application of existing instruments is planned within the research project. A further project objective is the development of a catalogue of measures for competence development on the one hand classified according to the type of measure (e.g. on-the-job, off-the-job) and on the other hand according to the competence which should be developed. It is planned not to limit the catalogue of measure to organized learning situations but also to support the informal development of competencies, e.g. by providing design recommendations for a work organization which supports learning. By comparing the existing competencies (current state profile) of the employee with the required competencies (target profile) of the role, a prediction concerning the selection of competence development measures will be possible in the case of significant differences between the target and the current state profiles.

\section{EVALUATION PROJECT}

The method which is presented is being evaluated within a concrete engineering project which serves as an evaluation project within the AerViCo-consortium.

The processing of the evaluation project takes place on 3 levels which are: project level, VC level and evaluation level (see figure 5).

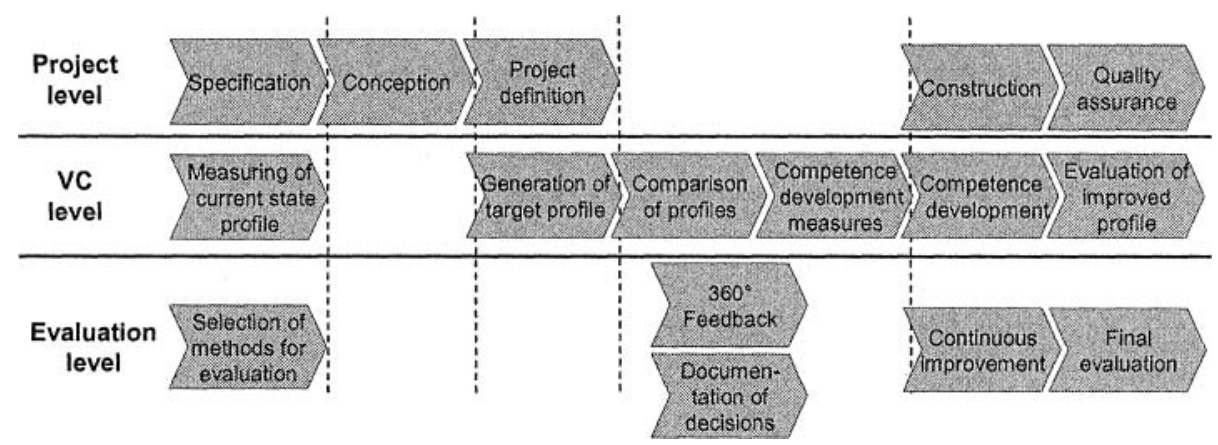

Fig. 5: Process model of the evaluation project

The project level is the conception and construction of one part of the mentioned engineering project - the development of a playground and entertainment area for children. 
On VC level the developed methods for competence profiling will come into operation in order to support the personnel placement within the consortium. In an early stage of the project the current state competence profiles of potential project team members will be measured using a commercial method of competence measurement. After the definition of the project, the presented method will be applied to generate the target competence profiles of the roles required within the project. By comparing the current state profiles with the target profiles the team members suited best for the requirements of the project will be chosen. If necessary measures for competence development will be derived from the comparison of profiles in order to further qualify the project team on the job.

The verification and evaluation of the target profiling instrument takes place on the evaluation level. By using special questionnaires and interview guidelines the effectiveness of the method for supporting the selection of team members and team qualification will be evaluated. The results of the evaluation (on the evaluation level) will be incorporated in a continuous and iterative further development.

\section{DISCUSSION AND OUTLOOK}

In this article a procedure to generate target competence profiles for roles and positions in a Virtual Company was presented which is based on the one hand on an allocation of competencies to activities and activity features and on the other hand on an allocation of activities and activity features to tasks (regarding a role or position). In order to measure the existing competencies of the employees involved in the cooperation (current state competence profile), existing tools of competence measurement will be used.

Currently the developed method is being applied in an engineering project in order to evaluate the practicability and, if necessary, to further improve the method. One of the next steps in the project progression will be the development and application of a catalogue of measures for competence development.

\section{ACKNOWLEDGMENTS}

The Project "AerViCo - Aerospace Virtual Company" is funded by the German Federal Ministry of Education and Research (grant number: 01HU0160 01HU0169). The other partners are: AIDA Development GmbH, Schwaebisch Hall, ALROUND e.V., Bonn, ANSYS Germany GmbH, Otterfing, Fraunhofer - Institut für Materialfluss und Logistik (IML), Dortmund, HEGGEMANN Aerospace AG, Büren, HTS GmbH, Coswig, IMA Materialforschung und Anwendungstechnik $\mathrm{GmbH}$, Dresden, MST Aerospace GmbH, Köln, ProTec-Recycling, Werne. 


\section{REFERENCES}

1. Erpenbeck, J. \& von Rosenstiel, L. 2002, Handbuch der Kompetenzmessung. Stuttgart: SchafferPoeschel.

2. Kabel, D. 2001, Entwicklung eines prozeBbasierten Effizienzmodells für Concurrent Engineering Teams. Dissertation RWTH Aachen, Aachen: Shaker-Verlag.

3. Killich, S., Peters, M.: The Interest of employees in Knowledge Sharing: A Theoretical Framework for the Integration of Motivation, Qualification and Organization for Knowledge Management in Networks. In: Luczak, H. ; Zink, K.J. (eds.): Human Factors in Organizational Design and Management - VII. Proceedings of the Seventh International Symposium on Human Factors in Organizational Design and Management held in Aachen, October 1-2, IEA Press, Santa Monica, CA, USA 2003, 373-378.

4. Odenthal, B. \& Peters, M. 2005, Specification Model for the Development and Operation of a Virtual Company in the Aerospace Industry, Proceedings of the PRO-VE'05 - 6th IFIP Working Conference on Virtual Enterprises held in Valencia (Spain). New York: Springer, 2005, 371-378.

5. Peters, M., Bernhard, J.: Virtuelle Zulieferkooperationen in der Luftfahrtindustrie - Entwicklung eines Konzeptes zum Aufbau und Betrieb Virtueller Unternehmen unter Berucksichtigung organisatorischer, technischer und personeller Faktoren. In: Unternehmen der Zukunft, Aachen, $5,2004,4 ; 3-4$.

6. Richter, G. 2001, Psychologische Bewertung von Arbeitsbedingungen. Bremerhaven: Wirtschaftsver1. NW, Verl. für neue Wiss.

7. Stahl, J. : Entwicklung einer Methode zur Integrierten Arbeitsgestaltung und Personalplanung im Rahmen von Concurrent Engineering. Dissertation RWTH Aachen, Aachen: Shaker-Verlag. 